\title{
Escala bidimensional para mensurar os custos de transação no varejo farmacêutico
}

\section{Adoption of a two-dimensional scale in the pharmaceutical retail transactions}

\author{
ADILSON ADERITO DA SILVA
}

\section{RESUMO}

O artigo está fundamentado na literatura da Teoria dos Custos de Transação (TCT) para propor e validar uma escala bidimensional composta por indicadores que permitiram captar a intensidade dos custos de transação ex ante e ex post percebidos pelos gestores nas transações do Varejo Farmacêutico. Os indicadores da escala refletiram os custos de análise, elaboração e adaptação contratual, além dos custos de coordenação, manutenção, monitoramento e guarda contra o oportunismo em situações de troca. A escala reuniu validade convergente, validade discriminante, consistência interna, confiabilidade e excelentes níveis de ajuste. A validação da escala, além de contribuir para o dimensionamento dos custos de transação, também permitirá testar empiricamente as relações desses custos com as dimensões analíticas propostas na TCT e seus impactos nas operações do setor estudado.

Palavras-chave: Custos de Transação, Validação de Escala, Varejo Farmacêutico

\section{Abstract}

The paper based on the literature of Transaction Cost Economics Theory (TCE) to propose and validate a two-dimensional scale composed of indicators that allowed capturing the intensity of ex-ante and ex-post transaction costs perceived managers in pharmaceutical retail transactions. The scale indicators reflected the costs of analysis, elaboration, and contractual adaptation, in addition to the costs of coordination, maintenance, monitoring, 
and custody against opportunism in exchange situations. The scale gathered convergent validity, discriminant validity, internal consistency, reliability, and excellent adjustment levels. The validation of the scale contributing to the dimensioning of transaction costs will also allow empirical testing of the relationships of these costs with the analytical dimensions proposed in TCE and its impacts on the operations of the studied sector Keywords: Transaction Costs, Scale Validation, Pharmaceutical Retail

\section{INTRODUÇÃo}

Ao longo das últimas décadas a Teoria dos Custos de Transação - TCT ganhou notoriedade por delinear a escolha de mecanismos eficientes de governança para economizar em custos de transação, os quais englobam despesas com a organização e coleta de informações; a negociação, formalização, execução e manutenção de contratos. Essa abordagem teórica foi estruturada por Oliver Williamson a partir da década de 70 e está fundamentada nos pressupostos comportamentais da racionalidade limitada e do comportamento oportunista de agentes econômicos.

A TCT defende que a escolha de mecanismos eficientes de governança pode ser influenciada por três dimensões analíticas: a especificidade dos ativos, caracterizada por investimentos idiossincráticos de difícil reaproveitamento outras atividades; a incerteza gerada por mudanças, escassez ou complexidade das informações e; pela frequência com que as transações são realizadas.

Inicialmente, Williamson propôs dois mecanismos polarizados de governança: o Mercado, governado por mecanismos de preço, e a Hierarquia caracterizada pelo fluxo de transações internas e verticalizadas. Entretanto, a TCT recebeu críticas por desconsiderar a eficácia das relações humanas no controle social e sua influência na adoção de outras formas ou estruturas de governança como as alianças, os acordos de cooperação, as trocas comerciais entre amigos, dentre outros relacionamentos baseados em confiança mútua e nas expectativas de ganhos futuros, construídas cuidadosamente ao longo das interações entre os parceiros de negociação (AXELROD, 1984; DYER, 1997; GRANOVETTER, 1985), assim como, pela pouca 
ênfase dada às vantagens e oportunidades advindas da cooperação interorganizacional para o desenvolvimento de habilidades competitivas integradas e à criação conjunta de valor (GHOSHAL; MORAN, 1996).

Essas críticas foram assimiladas por Williamson (1991) com a proposta de um modelo microanalítico que pauta a escolha de mecanismos de governança segundo o grau de especificidade dos ativos transacionados. Essa escolha se inicia com a forma mais flexível de coordenação pelo mecanismo de preços do mercado, em geral marcada por transações com baixa especificidade, passando pelas formas híbridas e estendendo-se às formas mais hierárquicas voltadas para a coordenação de recursos específicos com elevada propensão de oportunismo, formando assim um continuum de mecanismos de governança em grau crescente de integração vertical.

A importância da TCT é ressaltada pelos diversos estudos empreendidos na literatura nessas últimas décadas com o intuito de testar e validar seus pressupostos e dimensões, contudo, verifica-se um menor grau de atenção dado à mensuração desses custos pelos estudiosos dessa vertente teórica. Este estudo pretende contribuir com estudos futuros direcionando o seu foco para a mensuração dos custos de transação no Varejo Farmacêutico.

\subsection{Caracterização do Problema de Pesquisa}

Diversos estudos foram empreendidos para testar empiricamente a validade preditiva dos pressupostos e dimensões propostas na literatura da TCT. Skarmeas, Kats e Schlegelmilch (2002) testaram o impacto do oportunismo, da incerteza e dos investimentos em ativos específicos no grau de comprometimento entre importadores e fornecedores com o sucesso do relacionamento. Sampson (2004) analisou os custos do desalinhamento de mecanismos de governança em alianças para pesquisa e desenvolvimento. Carson, Madhok e Wu (2006) compararam eficácia das governanças contratual e relacional para coibir o oportunismo na coordenação de ativos específicos sob incerteza. Nygaard (2009) explorou as dimensões dos custos de transação e testou as predições da TCT quanto aos custos de negociação associados ao sistema de incentivos de um contrato bilateral no sistema norueguês de comercialização de combustíveis 
e evidenciaram a associação entre o controle vertical, o oportunismo e os custos de transação. Ruester e Zschille (2010) investigaram o impacto da estrutura de governança no desempenho de empresas. Marcher e Richman (2008) e Ruester (2010) evidenciaram a expressividade de estudos que utilizaram os ativos específicos e a incerteza como variáveis explicativas dos custos de transação.

Estudos empíricos com a fundamentação da TCT também têm sido desenvolvidos no Brasil. Lazzarini, Zylbersztajn e Takaki (1998) testaram o efeito das inovações contratuais no mercado futuro de boi gordo. Silva e Saes (2005) analisaram os fatores responsáveis pela coexistência de diferentes estruturas de governança sob reduzida especificidade dos ativos na cadeia produtiva do frango. Claro e Claro (2004) testaram a importância da confiança e o efeito dos investimentos em ativos específicos nos relacionamentos colaborativos com fornecedores. De Andrade et al. (2011) testaram a influência da confiança nos custos de transação. Silva e Brito $(2010 ; 2013)$ avaliaram o impacto da incerteza, da racionalidade limitada e da especificidade dos ativos no comportamento oportunista em cadeias de suprimento. Entretanto, esses estudos foram direcionados à análise preditiva das dimensões das transações e de seus pressupostos.

Barzel (1997) por sua vez, relacionou os custos de transação aos direitos de propriedade, contudo, autor concluiu que tal a mensuração seria difícil, cara e imprecisa devido à quantidade e à variabilidade dos atributos envolvidos de uma transação para outra. De igual forma, Zylberstjan (2010) argumentou que a mensuração dos custos de transação tem se mostrado uma tarefa mais difícil para se empreender do que testar hipóteses sobre o alinhamento dos mecanismos de governança às dimensões das transações ao nível micro ou às mudanças institucionais, ao nível macro.

Por outro lado, Furubotn e Richter (2000) atrelaram os custos de transação aos custos da utilização do mecanismo de preços do mercado, aos custos de gerenciamento, aos custos fixos e aos custos de transações políticas incorridos na lide com as mudanças institucionais. Grover e Malhotra (2003) desenvolveram um instrumento para mensurar os custos de transação baseado na percepção dos gestores quanto aos esforços associados com o desenvolvimento e monitoramento do desempenho do parceiro na transação; com a re- 
solução de possíveis problemas no relacionamento e a possibilidade de ocorrência do comportamento oportunista.

Nesta linha de atuação, Angelis et al. (2008) utilizaram os custos da utilização do Program Management Office - PMO como proxy dos custos de transação inerentes a coordenação e motivação na compra de sistemas de armamento pelo Departamento de Defesa, porém, não evidenciaram uma relação direta significativa. Enquanto, Barthélemy e Quélin (2006) investigaram os custos de transação advindos da complexidade dos contratos de terceirização mediante especificidade e incerteza utilizando indicadores que refletiam a proximidade dos parceiros com o núcleo dos negócios, os custos de mudança e os custos de adaptação e os resultados obtidos no estudo suportaram as predições da TCT.

No ambiente acadêmico brasileiro se destacam os estudos conduzidos por Caleman et al (2006) que ressaltam a efetiva necessidade de mensuração dos custos de transação como uma lacuna teórica a ser suprida. Zylberstjan e Graça (2003); Zylbersztajn, Faccioli e Frota Da Silveira (2007) e Zylberstjan (2010), apesar de apresentarem os problemas metodológicos para mensuração dos custos de transação, esses autores utilizaram como proxy de custos de transação ex ante os custos para se obter o direito legal para operar um novo negócio, ou seja, os custos de abertura de firmas.

Serigati e Azevedo (2014) propuseram uma ferramenta estatística combinando modelos de cointegração para mensurar indiretamente os custos de transação no mercado internacional de etanol e verificaram que o procedimento é potencialmente útil na avaliação de políticas para aumentar a eficiência do mercado. Enquanto, Kilinsky e Souza (2016) aplicaram o instrumento desenvolvido por Grover e Malhotra (2003) para mensurar a percepção de gestores sobre custos de transação em contratos de terceirização de processos em áreas de back-office operacional em uma instituição financeira.

Nogueira e Bataglia (2012) testaram a influência dos custos de transação e das competências organizacionais na escolha das estruturas de governança considerando os estágios iniciais da produção industrial. Bataglia et al. (2013) constataram que os custos de transação afetaram negativamente o desempenho das alianças e, que ao nível das rotinas das atividades da empresa com 
os parceiros, a capacidade relacional minimizou os efeitos dos custos de transação e elevou o desempenho das alianças. Entretanto, diferentemente dos estudos conduzidos na literatura de TCT, que utilizam as dimensões analíticas das transações para delinear a escolha de estruturas eficientes de governança com vistas a minimizar custos de transação, esses autores associaram os indicadores de incerteza, de especificidade dos ativos e frequência das negociações como proxy de custos de transação.

Diante da necessidade que se evidencia na literatura de TCT referente à mensuração dos custos de transação, o objetivo deste artigo é propor e validar uma escala bidimensional para estimar custos de transação ex ante e ex post no Varejo Farmacêutico. Para tal, estabeleceu-se como objetivos específicos: (1) caracterizar os custos de transação; (2) estabelecer indicadores que reflitam aos custos de análise e elaboração de contratos, custos de mudança e custos de adaptação contratual; (3) estimar os custos de transação percebidos pelos gestores que atuam no Varejo Farmacêutico.

\section{REFERENCIAL TEÓRICo}

Nesta seção se discorrerá a respeito da Teoria dos Custos de Transação e, mais, especificamente a respeito das definições de custos de transação adotadas no estudo.

\subsection{Teoria dos Custos de Transação}

Williamson (1975) verificou que após a celebração de contratos algumas ineficiências contratuais podem surgir devido à existência de assimetrias de informações nas relações bilaterais. Para lidar com essas ineficiências, a adaptação dos agentes às mudanças das condições ambientais ou contratuais é fundamental uma vez que todos os contratos em sua essência são incompletos, devido à incerteza dos eventos no ambiente ou pela limitação desses agentes para processar informações relevantes à transação a fim de prever todos os desdobramentos possíveis de uma relação contratual.

A Teoria dos Custos de Transação está embasada em dois pressupostos comportamentais: a racionalidade limitada e o comportamento oportunista. Quanto à racionalidade dos gestores Simon (1958) argumenta que a disposição das partes envolvidas na 
negociação à ação racional está restrita aos limites da capacidade humana em comunicar e processar informações relevantes, ou seja, os gestores agem com intenção racional, porém de forma limitada. Portanto, na elaboração de contratos torna-se difícil prever, com clareza, tanto as contingências do ambiente de negócios quanto àquelas relacionadas ao comportamento futuro dos parceiros de negociação, pois, independente de quaisquer pretensões dos agentes para uma ação racional, existem limitações naturais para capturar, processar e comunicar informações e, essas limitações, afetam os níveis de racionalidade desses agentes.

O comportamento oportunista, segundo pressuposto da TCT, é definido por Williamson (1985, p.47) como uma ação intencional “em que os agentes econômicos buscam os seus próprios interesses nas transações, agem em benefício próprio aproveitando-se de lacunas ou omissões contratuais em detrimento dos parceiros". Assim, nessa abordagem teórica, o oportunismo é visto como um traço comportamental inerente aos gestores envolvidos nas negociações o qual se manifesta pela manipulação estratégica da informação ou falseamento das intenções. Isso significa que, a priori, os gestores não hesitarão em mentir, trapacear, omitir e distorcer informações para alcançar seus objetivos.

Na conjugação da Racionalidade Limitada com o Comportamento Oportunista está essência da explicação da TCT para a emergência dos custos de transação, pois, são das lacunas, dos erros ou omissões contratuais não previstas ou antecipadas no contrato original, que resultam as dificuldades dos gestores para efetuar adaptações a fim de evitar as falhas de mercado e, por fim, os custos de transação na execução dos contratos. Então, a simples possibilidade de ocorrência do comportamento oportunista aumenta a incerteza nas negociações e dificulta a elaboração e a execução dos contratos (WILLIAMSON, 1979).

Dessa forma Teoria dos Custos de Transação busca descrever a firma não somente em termos de produção e transformação de matéria prima em bens de consumo, mas, principalmente em sua função da sua organização com vistas à escolha de estruturas de governança eficientes para minimizar custos de transação em suas operações. 


\subsection{Custos de transação}

Os custos de transação permeiam todas as etapas do processo produtivo e englobam as despesas com a organização e participação nas transferências do mercado. Coase $(1937 ; 1960)$ argumenta que os custos de transação decorrem da utilização do mecanismo de preços do mercado, envolvem os custos da coleta de informações relacionadas à busca de parceiros, avaliação, mapeamento dos atributos e vantagens dos produtos negociados; elaboração, monitoramento e execução dos contratos. Esses custos estão associados à descoberta de preços relevantes, à negociação e ao estabelecimento de contratos, ou seja, os custos dos recursos utilizados para a criação, manutenção, uso e mudança de instituições e organizações, portanto, são os custos para se movimentar o sistema econômico (ARROW, 1974).

Williamson (1985) também relacionou os custos de transação àqueles incorridos nas relações contratuais entre os agentes econômicos com vistas ao planejamento, adaptação e monitoramento das relações contratuais. Matthews (1986) os distinguem dos custos de produção, para o autor os custos de transação envolvem os custos de preparação de um contrato ex ante e de monitoramento ex post.

Cheung (1990) argumenta que os custos de transação são aqueles decorrentes da elaboração e negociação dos contratos; mensuração e fiscalização de direitos de propriedade; monitoramento do desempenho e organização da atividade produtiva. Enquanto Polski (2001) define esses custos como a soma dos custos associados com o engajamento em transações e atividades contratuais e, à semelhança de Matthews (1986), os distinguem dos custos de produção.

De acordo com Pilling, Crosby e Jackson (1994) os custos de transação são custos para desenvolver e manter uma relação de troca; monitorar o comportamento e proteger contra o oportunismo em uma situação de troca. O autor defende que os custos de desenvolvimento estão associados à elaboração de regras nas relações com os parceiros de transação, enquanto os custos de monitoramento lidam com os esforços para avaliar o desempenho do papel esperado dos parceiros. Angelis et al (2007) acrescentam aos custos de transação os custos de coordenação e motivação, os quais estão presentes em todos os acordos contratuais. 
Pode-se verificar que os autores, à semelhança de Williamson (1985), segmentaram os custos de transação em custos ex ante no sentido de buscar, preparar, negociar e proteger uma transação e custos de ex post com o monitoramento, ajuste e adaptação das transações em função de mudanças causadas por erros, omissões e alterações inesperadas.

Neste estudo, seguindo a lógica utilizada por Pilling, Crosby e Jackson (1994) os custos de transação serão entendidos com aqueles incorridos com o desenvolvimento, a coordenação, a manutenção, monitoramento do comportamento e guarda contra o oportunismo em uma situação de troca. Tal definição engloba os custos ex ante para se estabelecer o papel dos parceiros nas relações de transação; os custos com pesquisas para identificar opções, aquisição de informação oportuna, precisa e relevante para avaliar, negociar e escolher alternativas e redigir um contrato e os custos ex post com esforços para acessar, estabelecer o papel esperado do parceiro, monitoramento e avaliação do seu desempenho na transação e; custos para promover esforço produtivo, incentivos para investimentos a fim de dissuadir negociações improdutivas e comportamentos oportunistas.

Na próxima seção serão apresentados os procedimentos metodológicos utilizados no estudo e os procedimentos de coleta, tratamento e análise dos dados.

\section{Procedimentos Metodológicos}

Considerando a natureza subjetiva das variáveis latentes que refletem os custos de transação, as quais não podem ser mensuradas diretamente, para efetuar o processamento dos dados optou-se pela técnica multivariada de Modelagem em Equações Estruturais. Essa técnica permite estimar e avaliar simultaneamente as múltiplas relações de dependência e inter-relações entre variáveis latentes por meio da especificação de modelos estruturais, ou seja, "é uma técnica multivariada que combina aspectos de regressão múltipla e análise fatorial" (HAIR JR. et al., 2009, p. 466). O processamento dos dados se dará com o software EQS 6.2 pelo método Maximum Likelihood levando-se em consideração as recomendações de Bentler e Chou (1987) relativas ao processamento dos dados provenientes 
de escalas ordinais, uma vez que a escala Likert introduz algum grau de não-normalidade à distribuição devido a natureza discreta dos dados obtidos.

De acordo com Finney e Di Stefano (2006) e Bentler(1995) os dados ordinais com distribuições aproximadamente normais provenientes de escalas com no mínimo cinco categorias podem ser tratados como dados contínuos pelo método de estimação Maximum Likelihood ou com Maximum Likelihood Robust (ML ROBUST) que ajusta o modelo pela estatística Satorra-Bentler Scaled Qui-Quadrado $(S B \chi 2)$ e permite determinar estatísticas confiáveis e igualmente estáveis quando a amostra é relativamente pequena.

Um passo importante na validação de um instrumento de coleta de dados ou escala é estabelecer a correspondência entre a variável definida conceitualmente (construto) e o procedimento operacional utilizado para mensurá-lo (SCHWAB, 1980; RAYKOV, 2015; MACKENZIE; PODSAKOFF; PODSAKOFF, 2011). Nesse sentido, entende-se que a validade de construto se refere ao grau que um instrumento, mensurará verdadeiramente o construto que se intenta mensurar. Segundo Bagozzi (1981) e Mackenzie, Podsakoff, Podsakoff (2011) a validação de construto engloba cinco tipos de validades, a saber: 1) a validade de conteúdo; 2) a consistência interna (unidimensionalidade e confiabilidade); 3) a validade convergente; 4) a validade discriminante e, 5) a validade nomológica ou preditiva (que não será testada neste estudo em função de não se estabelecer a priori uma rede de relacionamentos com o construto Custos de Transação).

A validade de conteúdo é um tipo qualitativo de validação que torna claro o domínio e as dimensões de um determinado conceito (BOLLEN, 1989). Neste tipo de validação o pesquisador se apoia em estudos prévios ou na opinião de especialistas para julgar se os elementos inseridos no instrumento de coleta têm a representatividade suficiente para refletir os significados associados ao construto em estudo. Já a consistência interna está relacionada aos conceitos de unidimensionalidade e de confiabilidade. Pela unidimensionalidade se assegura que as variáveis observáveis estão inteiramente relacionadas com o construto subjacente em estudo, enquanto que pela confiabilidade, geralmente expressa em termos do coeficiente Alfa de Cronbach, se avalia a magnitude dos relacionamentos di- 
retos entre as variáveis observáveis e o construto (BOLLEN, 1989; CRONBACH, 1951; NUNNALLY; BERNSTEIN, 1994).

A validade convergente reflete o grau de concordância existente entre duas ou mais medidas utilizadas para mensurar o mesmo conceito, enquanto a validade discriminante representa o grau em que as medidas de distintos conceitos diferem entre si (BAGOZZI; PHILLIPS, 1982, p. 468-469). Uma forma sofisticada para se avaliar os efeitos do método e dos conceitos surgiu com a Análise Fatorial Confirmatória (AFC) que permite testar diretamente a extensão do verdadeiro relacionamento entre os conceitos, propostos a priori, a partir da análise da variância e do erro aleatório (BENTLLER; BONETT, 1980; KENNY, 1979; KENNY; KASHY, 1992; ; BROWN, 2014; RAYKOV, 2015; MACKENZIE; PODSAKOFF; PODSAKOFF, 2011).

Em tal processo se adota um modelo nulo ou básico como referência onde nenhum relacionamento é estabelecido entre os construtos ou entre os construtos e as variáveis. A seguir são propostos novos modelos para se efetuar comparações hierárquicas a partir dos parâmetros de ajustes gerados a cada rodada da AFC. A comparação é efetuada a partir dos valores gerados para as estatísticas Qui-Quadrado, cujas significâncias são verificadas de acordo com as variabilidades dos graus de liberdade a cada um dos modelos comparados. Uma diminuição significativa da estatística Qui-Quadrado indica melhoria no ajustamento do modelo e, se tal melhoria resultou da fixação de correlações perfeitas entre pares de construtos, então tais construtos não se discriminam, logo, o modelo não reúne validade discriminante (KENNY; KASHY; 1992). As verificações das validades convergente e discriminante dos construtos propostos neste estudo serão efetuadas utilizando-se o método da (AFC).

\subsection{Operacionalização dos construtos da pesquisa}

Não obstante as medidas diretas e objetivas de custos de transação sejam poucas, difíceis e custosas para se obter, segundo Williamson (1993, p.44) “isto é, algumas vezes, remediável. Para alguns casos, as medidas indiretas podem por vezes ser idealizadas". Seguindo esse raciocínio, Masten, Meehan e Snyder (1991) investigaram a escolha organizacional na indústria de construção naval americana a partir da elaboração de uma pesquisa survey que 
permitiu construir uma medida de custos de governança interna daquela organização.

Neste estudo, a elaboração do instrumento de pesquisa se deu mediante varredura na literatura em busca de definições e indicadores que refletissem o construto de interesse a qual se deu três etapas. A primeira, pautou-se pela validação de conteúdo, que definiu o construto de forma apropriada como sugerem Diamantopoulos e Winklhofer (2001), Nunnaly e Berstein (1994) e Raykov (2015). Dessa forma, na definição do construto de Custos de Transação e sua mensuração utilizou-se como ponto de partida os indicadores das escalas identificadas no referencial teórico. A segunda etapa pautou-se pela seleção dos indicadores que melhor se adequavam à realidade investigada e, finalmente, na terceira etapa, os indicadores escolhidos passaram por um processo de tradução e adequação à realidade brasileira e do setor de interesse desta pesquisa.

Os custos de transação são interpretados neste estudo como os custos decorrentes com o desenvolvimento, a coordenação, a manutenção, monitoramento do comportamento e guarda contra o oportunismo em uma situação de troca e está segmentado: em custos ex ante, para o desenvolvimento e manutenção de uma relação de troca (custos de obtenção de informação, elaboração, negociação e proteção de um acordo).

Já os custos ex post, relacionados ao monitoramento do comportamento na transação; ajustes e adaptação devido às mudanças na execução do contrato causadas por falhas, erros, omissões e alterações inesperadas; e proteção contra o oportunismo conforme definiram (WILLIAMSON, 1985, CUNHA, SAES, MAINVILLE, 2011; PILLING; CROSBY; JACKSON, 1994; TOMASSEN, 2004).

Os construtos de transação ex ante e ex post serão estimados a partir de indicadores, organizados em uma escala Likert, cujas variabilidades captarão e refletirão a percepção dos respondentes quanto ao grau dos custos de transação presentes no ambiente de negócios do setor de Varejo Farmacêutico. Os indicadores desses custos foram adaptados da escala validada por Tomassen (2004) a partir dos estudos realizados por Williamson (1985) e Pilling, Crosb e Jackson (1994) e Cunha, Saes e Mainville (2011). 
Quadro 1-Assertivas de Custos de Transação

\begin{tabular}{|c|c|c|}
\hline & Assertivas & Autores \\
\hline \multirow{4}{*}{ 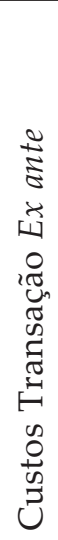 } & $\begin{array}{l}\text { O gasto de recursos com obtenção de informações } \\
\text { para se elaborar uma proposta de acordo comercial } \\
\text { com os nossos parceiros }\end{array}$ & \multirow{8}{*}{$\begin{array}{l}\text { William- } \\
\text { son (1985); } \\
\text { Cunha, } \\
\text { Saes e } \\
\text { Mainvil- } \\
\text { le (2011); } \\
\text { Pilling, } \\
\text { Crosby e } \\
\text { Jackson } \\
\text { (1994) e } \\
\text { Tomassen } \\
\text { (2004) }\end{array}$} \\
\hline & $\begin{array}{l}\text { O gasto de tempo para negociar acordos com os } \\
\text { parceiros comerciais antes da assinatura do contra- } \\
\text { to }\end{array}$ & \\
\hline & $\begin{array}{l}\text { O nível das perdas resultantes de falhas para se } \\
\text { obter um acordo inicial eficiente com os parceiros } \\
\text { comerciais }\end{array}$ & \\
\hline & $\begin{array}{l}\text { O montante de recursos gastos durante a negocia- } \\
\text { ção de acordos/elaboração de contratos comerciais } \\
\text { com os parceiros }\end{array}$ & \\
\hline \multirow{4}{*}{ 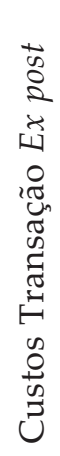 } & $\begin{array}{l}\text { O gasto de recursos para controlar a execução das } \\
\text { atividades do acordo comercial com os parceiros }\end{array}$ & \\
\hline & $\begin{array}{l}\text { O tempo gasto de tempo com os parceiros para re- } \\
\text { solver conflitos de interesses ao longo da execução } \\
\text { do acordo comercial }\end{array}$ & \\
\hline & $\begin{array}{l}\text { O gasto de tempo/recursos com adaptações contra- } \\
\text { tuais do acordo comercial ao longo de sua execu- } \\
\text { ção }\end{array}$ & \\
\hline & $\begin{array}{l}\text { Os gastos de recursos com incentivos à equipe ao } \\
\text { longo da execução do acordo comercial }\end{array}$ & \\
\hline
\end{tabular}

Fonte: elaborado pelo autor

Elaboradas as assertivas da escala, o próximo passo foi testar a validade de conteúdo dos indicadores mediante pré-teste com 30 profissionais do ramo para verificar os níveis de adequação, compreensibilidade, clareza e consistência interna.

A aplicação dos questionários estruturados foi realizada por empresa especializada em duas modalidades: in loco com visitas dos pesquisadores e, por autopreenchimento, via internet após consulta telefônica e envio de carta-convite quando se tratava de redes fora da Cidade de São Paulo.

O universo da pesquisa foi definido pelo conjunto de empresas que atuam no setor de Varejo da Indústria Farmacêutica Brasileira 
formado por redes de farmácias e farmácias independentes especializadas na comercialização de fármacos. Os respondentes-alvo da pesquisa foram profissionais que ocupavam cargos executivos ou gerenciais de nível médio ou superior, escolhidos por estarem mais aptos e qualificados para prover as informações pertinentes às questões da pesquisa.

\section{RESUltados da PESQUisa}

Apresentam-se nesta seção os resultados obtidos na pesquisa iniciando-se pela análise descritiva dos dados coletados, seguida pela análise de adequação, consistência interna dos indicadores e verificação das validades convergente e discriminante da escala proposta.

\subsection{Análise descritiva dos dados}

Foram enviadas cartas-convite para participar da pesquisa por meio de mensagens eletrônicas a 6548 gestores de farmácias cadastrados na base da ANVISA. Desses respondentes, houve o retorno de 173 que aceitaram participar da pesquisa, os quais foram contatados por telefone pela equipe responsável pela coleta dos dados que forneceu aos respondentes, informações mais detalhadas sobre $o$ estudo e seus objetivos. Após receberem as instruções eles passavam a responder o questionário eletrônico disponibilizado pela equipe.

As informações foram coletadas por meio da aplicação de questionário estruturado, respondido por 173 respondentes dos quais somente 122 profissionais assinalaram suas opiniões de forma completa e consistente, portanto, 122 questionários foram contabilizados como válidos e constituíram a amostra final utilizada no estudo.

Na Tabela 1, a seguir, são apresentadas as medidas descritivas resultantes do processamento dos dados coletados.

Tabela 1 - Distribuição dos respondentes por gênero e escolaridade

\begin{tabular}{|c|c|c|c|c|c|c|c|c|}
\hline & & \multicolumn{6}{|c|}{ Escolaridade } & \multirow[b]{2}{*}{ Total } \\
\hline & & Fundamental & Médio & Graduação & Pós-Grad & Mestrado & Doutorado & \\
\hline \multirow[t]{2}{*}{ Masculino } & Count & 1 & 18 & 45 & 14 & 0 & 0 & 78 \\
\hline & $\%$ of Total & $0,80 \%$ & $14,80 \%$ & $36,90 \%$ & $11,50 \%$ & $0 \%$ & $0 \%$ & $63,90 \%$ \\
\hline \multirow[t]{3}{*}{ Feminino } & Count & 0 & 3 & 26 & 12 & 2 & 1 & 44 \\
\hline & $\%$ of Total & $0 \%$ & $2,50 \%$ & $21,30 \%$ & $9,80 \%$ & $1,60 \%$ & $0,80 \%$ & $36,10 \%$ \\
\hline & Count & 1 & 21 & 71 & 26 & 2 & 1 & 122 \\
\hline Total & $\%$ of Total & $0,80 \%$ & $17,20 \%$ & $58,20 \%$ & $21,30 \%$ & $1,60 \%$ & $0,80 \%$ & $100 \%$ \\
\hline
\end{tabular}

Fonte: elaborada a partir dos dados da pesquisa 
Os dados indicam a predominância de respondentes do sexo masculino (63,9\%), dos quais 48,4\% possuem cursos de nível superior (Graduação/Pós-Graduação) em comparação com as respondentes do sexo feminino $(36,1 \%)$, dos quais $(33,5 \%)$ têm escolaridade em nível superior (Graduação/Pós-Grad/Mestrado/Doutorado) e apenas $(2,5 \%)$ delas cursaram o Ensino Médio contra (14,8\%) de homens nesse mesmo nível.

Tabela 2- Distribuição dos respondentes por gênero e cargo ocupado

\begin{tabular}{|c|c|c|c|c|c|c|c|c|c|c|c|}
\hline & & \multicolumn{9}{|c|}{ Cargo ocupado pelo respondente } & \multirow[b]{2}{*}{ Total } \\
\hline & & Proprietário & Diretor & Farmacêutico & $\begin{array}{c}\text { Gerente } \\
\text { Comercial }\end{array}$ & $\begin{array}{l}\text { Gerente } \\
\text { Compras }\end{array}$ & $\begin{array}{c}\text { Gerente } \\
\text { Operações }\end{array}$ & $\begin{array}{c}\text { Gerente } \\
\text { Geral }\end{array}$ & Supervisor & Outros & \\
\hline \multirow[t]{2}{*}{ Masculino } & Count & 34 & 1 & 8 & 17 & 3 & 5 & 6 & 0 & 4 & 78 \\
\hline & $\%$ of Total & $27,9 \%$ &, $8 \%$ & $6,6 \%$ & $13,9 \%$ & $2,5 \%$ & $4,1 \%$ & $4,9 \%$ & $0,0 \%$ & $3,3 \%$ & $63,9 \%$ \\
\hline \multirow[t]{4}{*}{ Feminino } & Count & 9 & 1 & 9 & 9 & 2 & 5 & 2 & 2 & 5 & 44 \\
\hline & $\%$ of Total & $7,4 \%$ &, $8 \%$ & $7,4 \%$ & $7,4 \%$ & $1,6 \%$ & $4,1 \%$ & $1,6 \%$ & $1,6 \%$ & $4,1 \%$ & $36,1 \%$ \\
\hline & Count & 43 & 2 & 17 & 26 & 5 & 10 & 8 & 2 & 9 & 122 \\
\hline & $\%$ of Total & $35,2 \%$ & $1,6 \%$ & $13,9 \%$ & $21,3 \%$ & $4,1 \%$ & $8,2 \%$ & $6,6 \%$ & $1,6 \%$ & $7,4 \%$ & $100,0 \%$ \\
\hline
\end{tabular}

Fonte: elaborada a partir dos dados da pesquisa

A mediana, a média e o desvio padrão da idade dos respondentes são, respectivamente, iguais a ), enquanto que os tempos de atuação na empresa e no setor foram, respectivamente, () e ().

Esses dados aliados à distribuição dos respondentes por cargo permitem verificar a adequação e a qualificação dos sujeitos para participar e fornecer informações relevantes a respeito dos construtos da pesquisa, ou seja, 111 respondentes (92,9\%) ocupam cargos de nível médio ou superior. Desses (38,8\%) são Proprietários/Diretores do ponto de venda; (40,2\%) são Gerentes e (13,9\%) Farmacêuticos. Verifica-se ainda que $(27,9 \%)$ dos proprietários são do sexo masculino e $(7,4 \%)$ são mulheres proprietárias. Na Tabela 3, a seguir, apresenta-se a caracterização das empresas segundo o número de pontos de vendas e o número de funcionários. 
Tabela 3 - Pontos de venda e número de funcionários

\begin{tabular}{lrrc}
\hline Pontos de Venda & Frequency & Percent & Cumulative \% \\
\hline 01 ponto & 66 & 54,1 & 54,1 \\
02 a 05 pontos & 23 & 18,9 & 73,0 \\
05 a 10 pontos & 5 & 4,1 & 77,0 \\
Acima 10 pontos & 28 & 23,0 & 100 \\
Total & 122 & 100 & \\
\hline \hline $\mathrm{N}^{\circ}$ funcionários & Frequency & Percent & Cumulative \% \\
\hline 01 até 50 & 85 & 69,7 & 69,7 \\
de 50 a 99 & 12 & 9,8 & 79,5 \\
de 100 a 500 & 8 & 6,6 & 86,1 \\
Acima de 500 & 17 & 13,9 & 100,0 \\
Total & 122 & 100,0 & \\
\hline
\end{tabular}

Fonte: elaborada com base nos dados da pesquisa

A amostra, em sua maioria (54,1\%), é constituída por empresas com apenas um ponto de venda, no entanto, as pequenas e médias redes de farmácias com dois a dez pontos de venda representam (22,9\%) da amostra e o restante (23\%) por empresas com dez ou mais pontos de venda. Quanto ao número de funcionários $(69,7 \%)$ das empresas empregam até 50 funcionários e (13\%) acima de 500 funcionários.

A seguir, apresenta-se na Tabela 4 a análise descritiva das assertivas elaboradas no estudo para estimar os custos de transação ex ante e ex post. Verifica-se que as assertivas de custos ex ante e ex post apresentaram medidas de tendências centrais moderadas.

Tabela 4 - Análise descritiva das assertivas utilizadas no estudo

\begin{tabular}{|c|c|c|c|c|c|c|}
\hline & ASSERTIVAS DO QURSTIONÁRIO & Meatan & Mode & Mean & Stal Dev & n \\
\hline \multirow{4}{*}{ 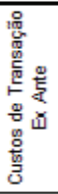 } & $\begin{array}{l}10 \text { gasto derecursos con a obtenção de irformaçōes para se estabelecer acondos } \\
\text { comerciais con } 05 \text { nossos parceiros é }\end{array}$ & 3,00 & 3 & 2,50 & 1,228 & 122 \\
\hline & $\begin{array}{l}20 \text { gasto de tempo paranegociar acordos com os parceiros comerciais antes da } \\
\text { ssinatura do contrato é: }\end{array}$ & 3,00 & 3 & 2,64 & 1.136 & 122 \\
\hline & $\begin{array}{l}\text { 3. O nivel das perdas resultantes de fathas para se obter un acordo inicial eficiente con } \\
\text { os oarceiros comerciais e: }\end{array}$ & 200 & 2 & 2,24 & 1,068 & 122 \\
\hline & 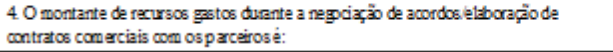 & 200 & 2 & 2,39 & 1,009 & 119 \\
\hline \multirow{4}{*}{ 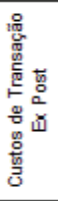 } & $\begin{array}{l}\text { 5. O gasto derecursos para controlar a eracuģo das atividades do acordo conercial } \\
\text { con os parceiros é: }\end{array}$ & 3,00 & 3 & 2,43 & 1,043 & 120 \\
\hline & $\begin{array}{l}\text { 6. O tenpo gsto de tempo com os parceiros para resclver conflitos de interesses ao } \\
\text { longo da erecucäo do acordo conercial é: }\end{array}$ & 3,00 & 3 & 2,70 & 1,130 & 121 \\
\hline & 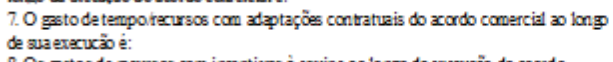 & 200 & 3 & 2,31 & 1,021 & 122 \\
\hline & $\begin{array}{l}\text { 8. Os gstos de secursos com incentivos à equipe ao longo da execução do acordo } \\
\text { comercial é: }\end{array}$ & 200 & 3 & 2,43 & 1,042 & 120 \\
\hline
\end{tabular}

Fonte: Os autores com base nos dados da pesquisa 


\subsection{Validação da escala de Custo de Transação}

Considerando a natureza subjetiva das variáveis latentes presentes no estudo, faz-se necessário verificar o grau de convergência e de consistência interna das assertivas propostas para cada construto conforme recomendam Mackenzie, Podsakoff e Podsakoff (2011); Raykov (2015) e Boateng, et al. (2018). Para tal, num primeiro momento, as assertivas do construto "Custo de Transação" foram processadas com a técnica estatística de Análise Fatorial, pelo método Principal Component Analyisis com rotação Varimax e critério do "eigenvalue maior que 1" para a extração dos fatores. Essa técnica é capaz de estimar as múltiplas relações de interdependência para testar a existência de convergência das assertivas propostas às variáveis latentes do estudo.

O processamento da análise fatorial se mostrou adequado pelo índice Kaiser Meyer Olkin( $\mathrm{KMO}=0,774$; sig=0,000) e pela medida MSA que apresentou valores no intervalo de [0671; 0,878], acima, portanto, do valores mínimos indicados na literatura para essas duas medidas (KMO>0,60 e MSA>0,50). Não obstante as assertivas apresentarem bons níveis de adequação pelo indicador MSA, as assertivas: "PERD-FAL - O nível das perdas resultantes de falhas para se obter um acordo inicial eficiente com os parceiros comerciais" e (INC_EQUP - Os gastos de recursos com incentivos à equipe ao longo da execução do acordo comercial) apresentaram baixos índices de comunalidade, respectivamente iguais a $(0,219$ e 0,181$)$. Nesta fase do estudo, essas assertivas foram mantidas e condicionadas à verificação de suas adequações nas análises subsequentes.

Tabela 5 - Análise Fatorial e Alpha de Cronbach para Custo de Transação

\begin{tabular}{|c|c|c|}
\hline & \multicolumn{2}{|c|}{ Component } \\
\hline & Ex Post & Ex Ante \\
\hline $\begin{array}{l}\text { 7. O gasto de tempo/recursos com adaptações contratuais do acordo comercial ao } \\
\text { longo de sua execução é: }\end{array}$ & 857 & \\
\hline $\begin{array}{l}\text { 6. O tempo gasto de tempo com os parceiros para resolver conflitos de interesses } \\
\text { ao longo da execução do acordo comercial é: }\end{array}$ & ,775 & \\
\hline $\begin{array}{l}\text { 8. Os gastos de recursos com incentivos à equipe ao longo da execução do acordo } \\
\text { comercial é: }\end{array}$ & ,622 & \\
\hline $\begin{array}{l}\text { 5. O gasto de recursos para controlar a execução das atividades do acordo comercial } \\
\text { com os parceiros é: }\end{array}$ &, 566 &, 513 \\
\hline $\begin{array}{l}\text { 4. O montante de recursos gastos durante a negociação de acordos/elaboração de } \\
\text { contratos comerciais com os parceiros é: }\end{array}$ & &, 758 \\
\hline $\begin{array}{l}\text { 3. O nível das perdas resultantes de falhas para se obter um acordo inicial eficiente } \\
\text { com os parceiros comerciais é: }\end{array}$ & &, 712 \\
\hline $\begin{array}{l}\text { 1. O gasto de recursos com a obtenção de informações para se estabelecer acordos } \\
\text { comerciais com os nossos parceiros é: }\end{array}$ & &, 680 \\
\hline $\begin{array}{l}\text { 2. O gasto de tempo para negociar acordos com os parceiros comerciais antes da } \\
\text { assinatura do contrato é: }\end{array}$ & &, 630 \\
\hline Extraction Method: Principal Component Analysis. & 28,52 & 28,47 \\
\hline a. Rotation converged in 3 iterations. &, 749 &, 704 \\
\hline
\end{tabular}

Fonte: dados da pesquisa 
Conforme se observa na Tabela 5, as assertivas de "Custo de Transação" convergiram em dois fatores, sendo o primeiro rotulado por "Ex post" e o segundo "Ex ante", com variância total explicada de 56,99\% e consistências internas (Alpha=0,749 e 0,704), ambas acima do valor mínimo de referência $($ Alpha $=0,60)$ proposto por Nunnally e Bernstein (1994).

A validade discriminante da escala de "Custo de Transação" foi testada pelo procedimento hierárquico onde se comparou os valores das estatísticas de ajustamento global (Qui-quadrado $\chi^{2}$; GFI e RMSEA) dos modelos (Modelo 0 - unidimensional; Modelo 1 - Bidimensional Independente; Modelo 2 - Bidimensional Correlacionado; Modelo 3 - Bidimensional de Segunda Ordem), (vide a Figura 2), assim como, pela análise dos índices de ajustamento comparativo (CFI e IFI) produzidos no processamento das assertivas com a Modelagem em Equações Estruturais - MEE (MACKENZIE; PODSAKOFF; PODSAKOFF, 2011; BROWN, 2014; BOATENG, et al., 2018)

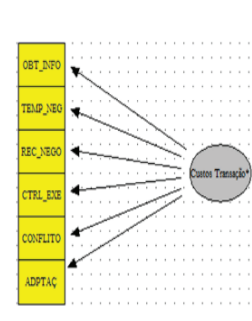

Modelo 0 - Unidimensional

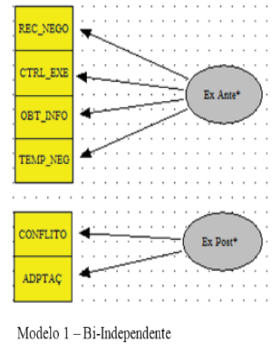

Modelo 1-Bi-Independente

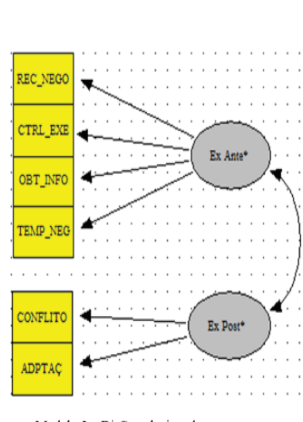

Modelo 2-Bi-Correlacionado

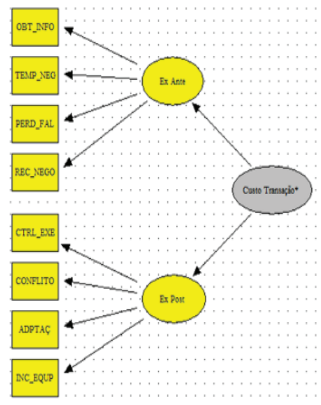

Modelo 3-CT de $2^{\text {a }}$ ordem

Figura 2 - Modelos utilizados no processamento do Método Hierárquico

Os resultados dos processamentos dos modelos estão sintetizados na Tabela 6 que apresentaram bons valores de ajuste, tanto pelo método Maximum Likelihood - ML quanto pelo método Maximum Likelihood Robusto - ML Robust. 
Tabela 6 - Validação do Construto custo de transação

\begin{tabular}{|c|c|c|c|c|c|c|c|c|c|c|c|c|}
\hline \multirow[b]{2}{*}{$\begin{array}{l}\text { MODE- } \\
\text { LOS }\end{array}$} & \multicolumn{7}{|c|}{ Maximum Likelihood - ML } & \multicolumn{4}{|c|}{\begin{tabular}{|l} 
Maximum Likelihood \\
Robust
\end{tabular}} & \multirow[b]{2}{*}{$\begin{array}{l}\text { RM- } \\
\text { SEA }\end{array}$} \\
\hline & $\chi^{2}$ & $\overline{G L}$ & $\mathrm{P}$ & CFI & IFI & GFI & $\begin{array}{l}\text { RM- } \\
\text { SEA }\end{array}$ & $\chi_{S B}^{2}$ & $\mathrm{P}$ & FI & IFI & \\
\hline Modelo 0 & 50,44 & 8 & 0,000 & 0,800 & $\overline{0,807}$ & 0,870 & 0,214 & 30,48 & 0,000 & 0,880 & 0,884 & 0,156 \\
\hline Modelo 1 & 50,72 & 7 & 0,000 & 0,794 & 0,802 & 0,887 & 0,232 & 20,98 & 0,003 & 0,925 & 0,928 & 0,131 \\
\hline Modelo 2 & 17,66 & $\overline{6}$ & 0,007 & 0,945 & 0,947 & 0,954 & 0,129 & 7,29 & 0,294 & 0,993 & 0,993 & 0,043 \\
\hline Modelo 3 & 17,65 & 4 & 0,001 & 0,936 & 0,939 & 0,954 & 0,172 & 4,05 & 0,395 & 1,000 & 1,000 & 0,010 \\
\hline
\end{tabular}

Fonte: sintetizado pelos autores

As diferenças entre as estatísticas Qui-Quadrado dos modelos (Modelo 0 e Modelo $1-\Delta \chi^{2}=0,279 ; \mathrm{gl}=1$ ) não foi significativa, portanto, não reuniu melhoria de ajuste na passagem de unidimensional para bidimensional independente. $\mathrm{J}$

Já em relação aos modelos independente e independente correlacionado (Modelo 1 e Modelo 2) houve diminuição significativa na estatística Qui-Quadrado $\left(\Delta \chi^{2}=-33,06 ; \mathrm{gl}=1\right)$ revelando que o Modelo 2 se mostra mais ajustado que o modelo unidimensional (Modelo 0) e o modelo bidimensional independente (Modelo 1). Já a diferença entre as estatísticas Qui-Quadrado do Modelo 2 e do Modelo 3 não foi significativa.

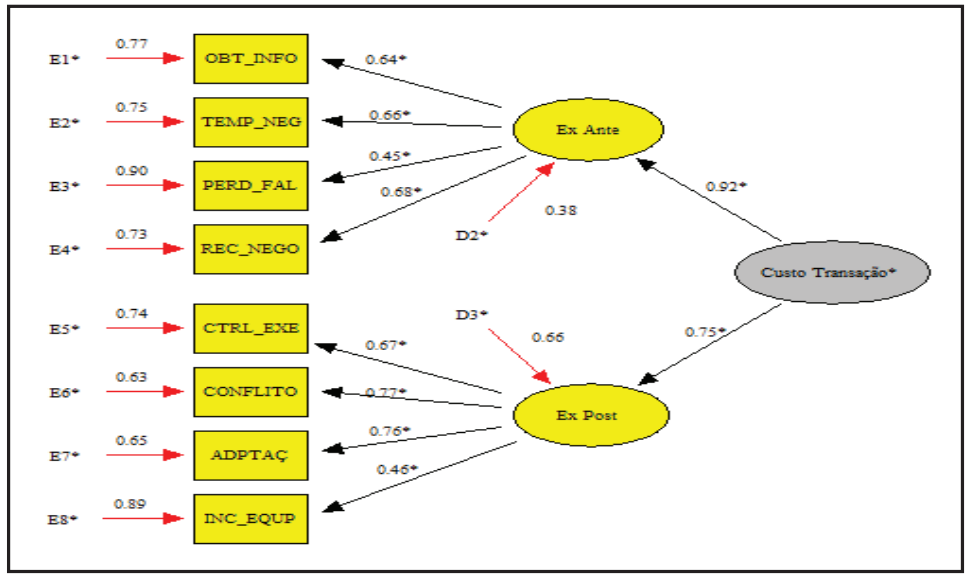

Figura 3 - Modelo de mensuração de Custo de Transação Fonte: dados da pesquisa 
Esses resultados revelam a natureza bidimensional do construto "Custo de Transação", ou seja, o construto reúne melhor nível de ajustamento quando disposto em dois fatores correlacionados, confirmando a validade discriminante dessa escala. O Modelo 3, ilustrado na Figura 3, apresentou cargas fatoriais significativas e boas consistências internas confirmando também a validade convergente dessa escala.

\section{CONSIDERAÇões FINAIS}

Esta pesquisa foi desenvolvida a partir das lacunas identificadas na literatura da TCT quanto à mensuração dos custos de transação. Geralmente os estudos nesta abordagem se limitam à análise das dimensões das transações e suas relações com a escolha de estruturas de governança para minimizar os custos de transação. Por outro lado, observou-se que as tentativas em se estabelecer medidas diretas e objetivas para mensurar os custos de transação são reduzidas, caras e imprecisas devido à quantidade de atributos e à variabilidade desses atributos de uma transação para outra. Não obstante, conforme observou Williamson (1993) isto pode ser remediado pela idealização de medidas indiretas.

Nesse sentido, a escala proposta para estimar os custos de transação, interpretados no estudo como os custos dispendidos com o desenvolvimento, a coordenação, a manutenção, o monitoramento do comportamento e guarda contra o comportamento oportunista em uma situação de troca, reuniu a validade de conteúdo, consistência interna, validade convergente e validade discriminante e bom nível de ajustamento, ressaltando a natureza bidimensional do construto "Custo de Transação", caracterizado por custos ex ante e custos ex post, conforme defendido na literatura da TCT. Dessa forma o objetivo do estudo foi plenamente alcançado e, com isso, foi possível verificar que um nível moderado de custos de transação foi percebido pelos gestores do setor do Varejo Farmacêutico que responderam à pesquisa.

À luz dos resultados obtidos, torna-se viável o desenvolvimento de outras pesquisas e estudos empíricos para testar a validade nomológica de modelos conjugando as dimensões analíticas e os pressupostos da TCT para avaliar a adequação das estruturas de 
governanças adotadas no setor com vistas a minimizar os custos de transação percebidos pelos gestores, ou seja, as relações entre os construtos de especificidade dos ativos, incerteza, frequência e suas interfaces com o oportunismos das partes nas negociações mediante o processamento simultâneo dessas relações com a Modelagem em Equações Estruturais.

\section{REFERÊNCIAS}

ANGELIS, D. I.; DILLARD, J.; FRANCK R.; MELESE. F. Measuring Transaction Costs in DoD Acquisition Programs. Naval Postgraduate School, Monterey, California, September, 2008. ARROW, K. The Limits of Organization. New York: Norton, 1974.

AXELROD, R.M. (1984), The evolution of cooperation, Basic Books, New York, 1984.

AZEVEDO, P. F. Nova Economia Institucional: referencial geral e aplicações para a agricultura. Agricultura em São Paulo. São Paulo: IEA, v. 47, n.1, p. 33-52, 2000

BARTHÉLEMY, J.; QUÉLIN, B. Complexity of Outsourcing Contracts and Ex post Transaction Costs: An Empirical Investigation. Journal of Management Studies, v.43, n.8, p.1775-1797, 2005

BARZEL, Y. Economic analysis of property rights. 2ed. Cambridge: Cambridge University Press, 1997.

BATAGLIA, W.; SILVA, A. A; KLEMENT, C. F.F.; SIMPLÍCIO, F. O papel da capacidade relacional na relação entre aliança estratégica e o desempenho da carteira de alianças. Relatório Científico Mackpesquisa. Universidade Presbiteriana Mackenzie. São Paulo, 2013.

BENTLER, P. M. EQS Structural equations program manual. Encino, CA: Multivarite Software, Inc. 1995.

BENTLER, P. M.; CHOU C. P.. Practical issues in structural modeling. Sociological Methods and Research. .v.16, n.1, p-78-117, 1987.

BOATENG, Godfred O. et al. Best practices for developing and validating scales for health, social, and behavioral research: a primer. Frontiers in public health, v. 6, 2018.

BROWN, M.W. Confirmatory Factor Analysis for Applied Research. New York, NY: Guildford Press, 2014.

CALEMAN, S. M. Q; SPROESSER, R. L.; LIMA, D. O. TREDEZIN, C. A. O. Mecanismos de Governança em Sistemas Agroalimentares: um enfoque na mensuração dos custos de transação. Revista de Economia e Agronegócio, v.4, n. 2, 2006.

CARSON, S. J.; MADHOK, A.; WU, T. Uncertainty, Opportunism, and Governance: The Effects of Volatility and Ambiguity on Formal and Relational Contracting. The Academy of Management Journal, v.49, n. 5, p. 1058-1077, 2006. 
CLARO, D.P; CLARO, P.B.O. Gerenciando Relacionamentos Colaborativos com Fornecedores. Revista de Administração de Empresas, vol. 44, n.4, p.68-79, 2004.

COASE, R. The nature of the firm. Economica, v.4, p. 386-405, 1937.

COASE, R. The problem of social costs. Journal of Law and Economics, 3 pp. 1-44, 1960.

CUNHA, Christiano França; SAES, Maria Sylvia Macchione; MAINVILLE, Denise Yvonne. Análise da complexidade nas estruturas de governança entre supermercados e produtores agrícolas convencionais e orgânicos no Brasil e nos Estados Unidos: a influência do custo de transação e de mensuração. Revista de Administração, v. 48, n. 2, p. 341-358, 2013.

DE ANDRADE, Carlos Henrique Mascarenhas et al. A Relação entre Confiança e Custos de Transação em Relacionamentos Interorganizacionais/The Relation between Trust and Transaction Costs in Interorganizational Relationships. Revista de Administração Contemporânea, v. 15, n. 4, p. $608,2011$.

DIAMANTOPOULOS, A., AND WINKLHOFER, H. M. Index Construction with Formative Indicators: An Alternative to Scale Development. Journal of Marketing Research (38:2), pp. 269-277, 2001.

DYER, J. H. Effective interim collaboration: how firms minimize transaction costs and maximise transaction value. Strategic Management Journal, 18: 535-556. 1996. doi:10.1002/ (SICI)1097-0266(199708)18:7<535::AID-SMJ885>3.0.CO;2-Z

FARINA, E. M. M. Q.; AZEVEDO, P. F. A.; SAES, M. S. M.Competitividade: Mercado, Estado e Organizações. São Paulo: Editora Singular, 1997.

FINNEY, S. J.; DiSTEFANO, C. Nonnormal and categorical data in structural equation models. In G.R. Hancock \& R.O. Mueller (Eds.). A second course in structural equation modeling, p. 269-314. Greenwich, 2006.

FURUBOTN, E.; RICHTER, R. Instituitions and economic theory. The Contribution of the New Institutional Economics. Michigan Press, 2000. p.556.Goldberg, V.P., Erickson, J.R., 1987. Quantity and price adjustment in long-term contracts: a case study of petroleum coke 30, 369-398 GHOSHAL, Sumantra; MORAN, Peter. Bad for practice: A critique of the transaction cost theory. Academy of management Review, v. 21, n. 1, p. 13-47, 1996.

GRANOVETTER, M. Economic action and social structure: The problem of embeddedness. Amer. Journal Sociology v.91, p.481-510, 1985.

GROVER, V.; MALHOTRA, M.K. Transaction cost framework in operations and supply chain management research: theory and measurement. Journal of Operations Management, 21 (4), 457-473, 2003.

HAIR JR. J. F.; ANDERSON, R. E.; TATHAM, R. L.; BLACK, M. C. Análise multivariada de dados. 6. ed. Porto Alegre: Bookman, 2009. 
HOFFMANN, Werner H.; NEUMANN, Kerstin; SPECKBACHER, Gerhard, The Effect of Interorganizational Trust on Make-or-Cooperate Decisions: Disentangling Opportunism-Dependent and Opportunism-Independent Effects of Trust. European Management Review, Vol. 7, Issue 2, pp. 101-115, 2010.

KILINSKY, Pedro I., SOUZA, Rodrigo P. Percepções sobre Custos de Transação em Contratos de Terceirização: Estudo Aplicado em uma Instituição Financeira de Varejo. In: Anais do XL Encontro da ANPAD 2016. Costa do Sauípe, 2016.

LAZZARINI, S. G.; ZYLBERSZTAJN, D.; TAKAKI, F. S. Inovações contratuais em mercados futuros: o caso do boi gordo na BM\&F. Revista de Administração Contemporânea, v. 2, n. 3, p. 07-26, 1998. Disponível em: http://dx.doi.org/10.1590/S1415-65551998000300002. Acesso em: 30/08/2013.

MACHER, J. T.; RICHMAN, B. D. Transaction cost economics: an assessment of empirical research in the social sciences. Business and Politics, v.10, n.1, p.1-63, 2008.

MACKENZIE, S. B.; PODSAKOFF, P. M.; PODSAKOFF, N. P. Construct measurement and validation procedures in MIS and behavioral research: integrating new and existing techniques. MIS Quarterly, v. 35, n. 2, p. 293-334, 2011.

MASTEN, S. E, MEEHAN, J. JR.; SNYDER, E. A.The Costs of Organization. Journal of Law, Economics, and Organization, 7(1): 1-25, 1991.

MATTHEWS, Robin CO. The economics of institutions and the sources of growth. The Economic Journal, v. 96, n. 384, p. 903-918, 1986.

NYGAARD, A. Measuring Transaction Costs in Plural Formed Marketing Channels: an empirical investigation of franchise units in the oil industry. Saarbrücken, VDM Publishing, 2009.

NOGUEIRA, A. C. L.; BATAGLIA, W. Transaction Costs and Organizational Competences: Explaining the Governance Structuring Stage. Journal of Technology Management \& Innovation, v.7, n.1, 2012.

NUNNAlly, J. C.; BERNSTEIN, I. H. Psychometric Theory. 3rd ed., McGraw-Hill Inc., 1994.

PILLING, B. K., CROSBY, L. A.; JACKSON, D.W. Relational Bonds in Industrial Exchange: An Experimental Test of the Transaction Cost Economic Framework. Journal of Business Research, v.30, p. 237-51, 1994.

RAYKOV T. Scale Construction and Development. Lecture Notes. Measurement and Quantitative Methods. East Lansing, MI: Michigan State University, 2015.

RUESTER, S.; ZSCHILLE, M. The impact of Governance Structure on firm Performance - an Application to the German Water Distriution Sectos. Utilities Policy, v. 18, n.3, p. 154-162, 2010 SAMPSON, Rachelle C. The cost of misaligned governance in R\&D alliances. Journal of Law, Economics, and Organization, v. 20, n. 2, p. 484-526, 2004. 
SERIGATI, F. ; AZEVEDO, P. F. . How to Indirectly Measure Market Transaction Costs. In: 18th annual ISNIE Conference, 2014, Durham-NC. 18th annual ISNIE Conference. Durham: ISNIE, 2014. v. 1. p. 1-35.

SILVA, A. A. Integração Vertical em Cadeias de Suprimentos e os Pressupostos da Teoria dos Custos de Transação: Um Teste Empírico. Tese de Doutorado (Administração de Empresas) Universidade Presbiteriana Mackenzie. São Paulo, 2009

SILVA, A. A.; BRITO, E. P. Z. Incerteza, Racionalidade Limitada e Comportamento Oportunista: Um Estudo Na Indústria Brasileira. In: XXXIV Encontro da Anpad- Enanpad 2010. Rio de Janeiro, 2010.

SILVA, A. A.; BRITO, E. P. Incerteza, racionalidade limitada e comportamento oportunista: um estudo na indústria brasileira. RAM, Rev. Adm. Mackenzie[online]. 2013, vol.14, n.1, pp. 176-201. ISSN 1678-6971. http://dx.doi.org/10.1590/S1678-69712013000100008.

SILVA, C. L.; SAES, M. S. M. A Questão da Coexistência de Estruturas de Governança na Economia dos Custos de Transação: Evidências Empíricas Na Avicultura De Corte Paranaense. Revista de Administração Mackenzie, v. 6, n. 3, 2005.

SIMON, H. Administrative behavior. 2ed. New York: Macmillan, 1958.

SKARMEAS, D.; KATSIKEAS, C.S.; SCHLEGELMILCH, B. B. Drivers of commitment and its impact on performance in cross-cultural buyers-Seller Relationships: The importer's Perspective. Journal of International Business Studies. v.33, n.4, 2002.

TOMASSEN, Sverre. The Effects of Transaction Costs on the Performance of Foreign Direct Investments. 2004. Tese de Doutorado. Norwegian School of Management.

WILLIAMSON, O.E. Markets and Hierarchies. New York: Free Press, 1975.

WILLIAMSON, O. E. Transaction-Cost Economics: The Governance of Contractual Relations. Journal of Law and Economics, v.22, p.233-261, 1979. In: The Transaction Cost Economics Project: The Theory and Practice of the Governance of Contractual Relations. Northampton, MA. Edward Elgar Publishing, 2013.

WILLIAMSON, O. E. The Economic Institutions of Capitalism. N.Y.: Free Press, 1985.

WILLIAMSON, O.E. Comparative economic organization: The analysis of discrete structural alternatives. Administrative Science Quarterly, v.36, p.269-296, 1991. In: The Transaction Cost Economics Project: The Theory and Practice of the Governance of Contractual Relations. Northampton, MA. Edward Elgar Publishing, 2013.

WILLIAMSON, O. The Economic Analysis of Institutions and Organisations - in General and with Respect to Country Studies. OECD Economics Department Working Papers, No. 133, OECD Publishing, Paris, 1993. DOI: http://dx.doi.org/10.1787/802045548530

ZYLBERSTAJAN, D. Entry costs and quality of business environment: a critical analysis. Revista Administração Mackenzie, v. 11, n.5, p. 151-161, 2010 
ZILBERSZTAJN, D.; GRAÇA, C. T. Costos de formalización de las empresas: medición de los costos de transación em Brasil. Revista de Economia Institucional, Bogotá, v. 5, n. 9, p. 146-165, 2003.

ZOLLO, M.; REUER, J. J.; SINGH, H. Interorganizational routines and performance in strategic alliances. Organization Science, v. 13, n. 6, p. 701-713, 2002.

WANG, Ning. Measuring transaction costs: an incomplete survey. Ronald Coase Institute, Working Paper, v. 2, 2003.

Recebido em: 5-12-2019

Aprovado em: 17-12-2020

Avaliado pelo sistema double blind review.

Disponível em http://mjs.metodista.br/index.php/roc 